\title{
Calculation and 3D analyses of ERR in the band crack front contained in a rectangular plate made of multilayered material
}

https://doi.org/10.1515/chem-2018-0057

received January 22, 2018; accepted March 5, 2018.

Abstract: An investigation into the values of the Energy Release Rate (ERR) at the band crack's front in the rectangular plate made of multilayered composite material is carried out for the opening mode. The corresponding boundary-value problem is modelled by using threedimensional linear theory and solved numerically by using 3D FEM (Three Dimensional Finite Element Method). The main purpose of the current investigation is to study the influence of mechanical and geometrical parameters on the Energy Release Rate (ERR) at this crack front. The numerical results related to the ERR, and the effect of the mechanical and other problem parameters on the ERR are presented and discussed.

Keywords: Energy Release Rate (ERR); band crack; 3D FEM (Three Dimensional Finite Element Method); rectangular plate.

\section{Introduction}

Since material defects such as cracks have a significant role in the processes of fractures, fracture mechanics investigates the mechanical behavior of fracture parameters (Stress Intensity Factors (SIF) or Energy Release Rates (ERR)) from various aspects such as load, material property and crack length. From recent research into this area, it can be seen that many of them have focused on determining the effects of anisotropy and other problem parameters on the SIF or ERR within the framework of the two-dimensional (2D) problem formulation [1-12]. Up to

*Corresponding author: Arzu Turan Dincel, Mathematical Engineering Department, Yildiz Technical University, 34220, Istanbul-Turkey, E-mail: artur@yildiz.edu.tr

Surkay D. Akbarov: Mechanical Engineering Department, Yildiz Technical University, 34349, Istanbul-Turkey now, there have not been any investigations regarding the corresponding 3D macro-crack problems for anisotropic bodies.

In connection with this, the purpose of this current paper is to investigate the effects of anisotropic properties of the plate material and other problem parameters on the ERR in the macro-band crack contained in an anisotropic plate.

\section{Problem Formulation and Solution Method}

Let usconsider athick rectangular plate(Figure1)occupying the domain $\Omega=\left\{0 \leq x_{1} \leq \ell_{1}, 0 \leq x_{2} \leq h, 0 \leq x_{3} \leq \ell_{3}\right\}$ in the Cartesian Coordinate system $O x_{1} x_{2} x_{3}$, which contains a band crack located in the region $\Omega^{\prime}=\left\{\left(\ell_{1} / 2-\ell_{0} / 2\right) \leq x_{1} \leq\left(\ell_{1} / 2+\ell_{0} / 2\right), x_{2}=h_{c} \pm 0,0 \leq x_{3} \leq \ell_{3}\right\}$. Assume that on the crack's edge planes, the opening uniformly distributed normal forces with intensity $\mathrm{p}$ act.

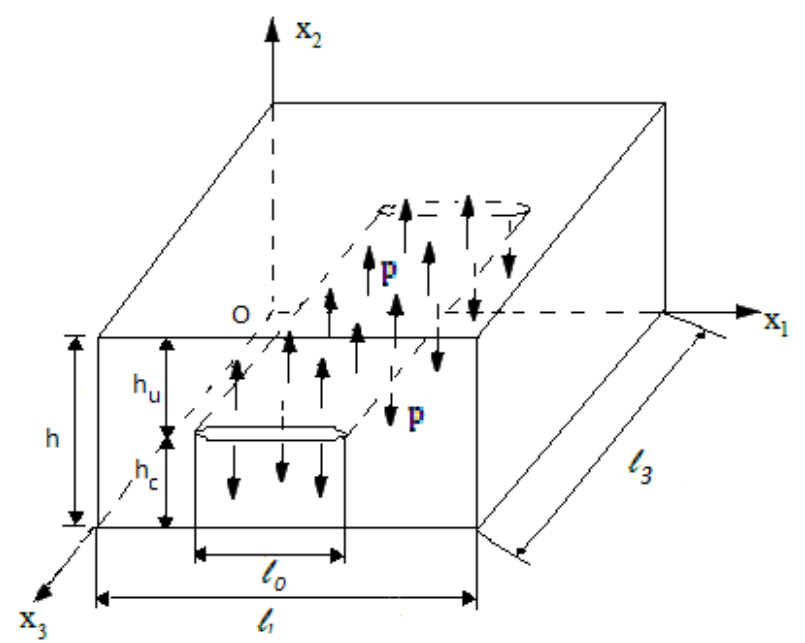

Figure 1: Geometry of a rectangular plate with a band crack. 
The field equations and boundary conditions for the case under consideration are:

$$
\begin{aligned}
& \frac{\partial \sigma_{i j}}{\partial x_{i}}=0, \sigma_{i i}=A_{i j} \varepsilon_{j j}, A_{i j}=A_{j i}, \sigma_{i j}=2 \mu_{i j} \varepsilon_{i j}, \text { at } i \neq j, \varepsilon_{i j}=\frac{1}{2}\left(\frac{\partial u_{i}}{\partial x_{j}}+\frac{\partial u_{j}}{\partial x_{i}}\right), \\
& \left.u_{2}\right|_{x_{1}=0}=\left.u_{2}\right|_{x_{1}=\ell_{1}}=0,\left.u_{2}\right|_{x_{3}=0}=\left.u_{2}\right|_{x_{3}=\ell_{3}}=0,\left.\sigma_{1 k}\right|_{x_{1}=0}=\left.\sigma_{1 k}\right|_{x_{1}=\ell_{1}}= \\
& =0,\left.\sigma_{3 k}\right|_{x_{3}=0}=\left.\sigma_{3 k}\right|_{x_{3}=\ell_{3}}=0, \\
& \left.\sigma_{2 i}\right|_{x_{2}=0}=0,\left.\sigma_{2 i}\right|_{x_{2}=h}=0,\left.\sigma_{2 i}\right|_{\Omega^{\prime}}=-p \delta_{i}^{2}, \quad i, j=1,2,3 ; k=1,3 .
\end{aligned}
$$

To solve the foregoing problem (1), we employ the 3D FEM and for this purpose we introduce the following functional:

$$
\begin{aligned}
& \Pi=\frac{1}{2} \iiint_{\Omega-\Omega^{\prime}}\left(\sigma_{11} \varepsilon_{11}+2 \sigma_{12} \varepsilon_{12}+2 \sigma_{13} \varepsilon_{13}+2 \sigma_{23} \varepsilon_{23}+\sigma_{22} \varepsilon_{22}+\sigma_{33} \varepsilon_{33}\right) d \Omega \\
& -\left.\int_{0}^{\ell_{3}} \int_{0}^{\ell_{1}} p u_{2}\right|_{x_{2}=h} d x_{1} d x_{3}-\left.\int_{0}^{\ell_{3}} \int_{0}^{\ell_{1}} p u_{2}\right|_{x_{2}=0} d x_{1} d x_{3}
\end{aligned}
$$

Using the Ritz technique [13], $\delta \Pi=0$, we obtain the equilibrium equation and boundary conditions in Eq. (1). In this way, the validity of the functional (2) for the FEM modelling of the considered problem is proven.

In these cases, the solution domain $\Omega$ is divided into a finite number of finite elements, which are selected as standard rectangular prisms (bricks) with eight nodes. Selection of the number of degrees of freedom (NDOF) is determined from the requirements that the boundary conditions should be satisfied with very high accuracy and that the numerical results obtained for various NDOFs should converge.

After consideration of the FEM modelling, the ERR (denoted by $\gamma$ ) is calculated by using the expression:

$$
\gamma \approx \frac{1}{2} \frac{U\left(S_{c}+\Delta S_{c}\left(s / \ell_{1}\right)\right)-U\left(S_{c}\right)}{\Delta S_{c}\left(s / \ell_{1}\right)}
$$

In Eq. (3), $U$ is the strain energy, $S_{c}$ shows the area of the crack's edge surface, $\Delta S_{c}$ is the increment of the crack's edge area, and $s / \ell_{1}=\left(0.5 \ell_{3}-x_{3}\right) / \ell_{1}$. In the solution procedure, the values of $\Delta S_{c}$ are selected regarding convergence of the numerical results obtained for $\gamma$.

Ethical approval: The conducted research is not related to either human or animals use.

\section{Numerical Results}

We assume that the plate is made of multilayered composite material consisting of alternating layers of two isotropic homogeneous materials parallel to the $O x_{1} x_{3}$ plane and introduce the following notation for the matrix and the reinforcing layers: $E_{1}$ and $E_{2}$ are the Young's moduli; $\nu_{1}$ and $\nu_{2}$ are the Poisson ratios; and $\eta_{1}$ and $\eta_{2}$ are the concentrations of the constituents. For the selected composite material, the effective anisotropic material constants $\mathrm{A}_{\mathrm{ij}}$ are determined according to the expressions given in [14].

The numerical investigations are performed for the case where $\eta_{2}=1-\eta_{1}=0.5, \nu_{2}=\nu_{1}=0.3$ and $\mathrm{h} / \ell=0.20$. Since the plate is symmetric with respect to the planes $x_{1}=\ell_{1} / 2$ and $x_{3}=\ell_{3} / 2$, then FEM solutions are obtained in a quarter part of the domain. This domain was divided into 30,12 , and 30 brick elements along the $\mathrm{Ox}_{1} \mathrm{x}_{2} \mathrm{x}_{3}$ axes.

Before obtaining numerical results, the PC programs composed and used by the authors, were tested with the known results given in [15].

Table 1 shows the values of $K_{I} / K_{I \infty}$ and $K_{I}^{S} / K_{I \infty}$ (where $K_{I \infty}=p \sqrt{\pi \ell_{0}}$ ) for various values of the ratios $\ell_{0} / \ell_{1}, \ell_{0} / h$ and $\ell_{3} / \ell_{1}$. Note that in Table 1, the values of $K_{I} / K_{I \infty}$ and $K_{I}^{S} / K_{I \infty}$ are SIF of Mode I; $K_{I} / K_{I \infty}$ is relevant to the plane strain state; and $K_{I}^{S} / K_{I \infty}$ is calculated by using exact solution for an infinite plate, for the approximate series as detailed in the handbook [15]. In this case, based on mechanical considerations, with increasing of the ratio $\ell_{3} / \ell_{1}$, $K_{I}\left(=\sqrt{\gamma /\left(1-\nu^{2}\right) \pi \ell_{0}}\right)$ at $s / \ell_{1}=0$ must approach the corresponding values obtained in [15] for the plane strain state. This prediction is confirmed by the data given in Table 1. Moreover, our results agree with the mechanical consideration according to which the values of $K_{I} / K_{I \infty}$ must approach 1 with increasing of the ratios $\ell_{3} / \ell_{1}$ and decreasing of the ratios $\ell_{0} / \ell_{1}$ and $\ell_{0} / h$. This comparison shows good agreement and confirms the validity of the algorithm and programs used in the present investigations.

Now, we analyze the numerical results which illustrate the influence of the problem parameters on the values of the ERR. Table 2 shows the influence of the ratios $s / \ell_{1}$ and $E_{2} / E_{1}$ on the ERR obtained in the case where $\ell_{0} / 2 \ell_{1}=0.15$ and $h_{u} / \ell_{1}=0.1$ (i.e. $h_{u}=h / 2$ ). It follows from Table 2 , as can be predicted, that the absolute maximum value of the ERR occurs at $s / \ell_{1}=0$ and the values of the ERR monotonically decrease with $s / \ell_{1}$. Moreover, it follows from Table 2, that the magnitude of the 
Table 1: The values of SIF at $s / \ell_{1}=0$ obtained for various values of the ratio $\ell_{3} / \ell_{1}$ in the case where the material of the plate is isotropic.

\begin{tabular}{|c|c|c|c|c|c|c|c|c|}
\hline \multirow[t]{2}{*}{$\ell_{0} / \ell_{1}$} & \multirow[t]{2}{*}{$\ell_{0} / h$} & \multicolumn{7}{|l|}{$\ell_{3} / \ell_{1}$} \\
\hline & & 1 & 5 & 10 & 15 & 20 & 50 & $K_{I}^{S} / K_{I \infty}[15]$ \\
\hline 0.080 & 0.80 & 0.9761 & 1.1771 & 1.2000 & 1.2029 & 1.2036 & 1.2356 & 1.2406 \\
\hline 0.075 & 0.75 & 0.9742 & 1.1720 & 1.1922 & 1.1943 & 1.1947 & 1.1948 & 1.2009 \\
\hline 0.060 & 0.60 & 0.9278 & 1.1129 & 1.1304 & 1.1329 & 1.1335 & 1.1341 & 1.1444 \\
\hline 0.050 & 0.50 & 0.8906 & 1.0658 & 1.0871 & 1.0909 & 1.0920 & 1.0931 & 1.0931 \\
\hline
\end{tabular}

Table 2: The influence of the ratio $E_{2} / E_{1}$ on the values of the ERR in the various $s / \ell_{1}$ in the case where $\ell_{0} / 2 \ell_{1}=0.15$ and $\ell_{3} / \ell_{1}=1$.

\begin{tabular}{llllllll}
\hline$E_{2} / E_{1}$ & $S / \ell_{1}$ & & & & & \\
& $\mathbf{0}$ & $\mathbf{0 . 1}$ & $\mathbf{0 . 1 3 3}$ & $\mathbf{0 . 1 6 6}$ & $\mathbf{0 . 2}$ & $\mathbf{0 . 3}$ & $\mathbf{0 . 4}$ \\
\hline 0.2 & 9.1266 & 9.0827 & 9.0355 & 8.9563 & 8.8266 & 7.8699 & 4.9238 \\
0.5 & 4.9865 & 4.9829 & 4.9677 & 4.9390 & 4.8583 & 4.2081 & 2.3733 \\
1 & 3.3233 & 3.3271 & 3.3212 & 3.3011 & 3.2564 & 2.8187 & 1.5627 \\
\hline
\end{tabular}

Table 3: The influence of the ratio $E_{2} / E_{1}$ on the values of the ERR in the various $\ell_{3} / \ell_{1}$ in the case where $\ell_{0} / 2 \ell_{1}=0.15$ and $s / \ell_{1}=0$

\begin{tabular}{llllll}
\hline $\boldsymbol{E}_{2} / \boldsymbol{E}_{1}$ & $\ell_{3} / \ell_{1}$ & & & & \\
& $\mathbf{0 . 2}$ & $\mathbf{0 . 5}$ & $\mathbf{1}$ & $\mathbf{1 . 5}$ & $\mathbf{2}$ \\
\hline 0.2 & 0.9391 & 6.4516 & 9.1266 & 9.8204 & 10.3322 \\
0.5 & 0.3087 & 3.2938 & 4.9865 & 5.4505 & 5.8451 \\
1 & 0.1883 & 2.1924 & 3.3233 & 3.6487 & 3.9346 \\
\hline
\end{tabular}

Table 4: The influence of the ratio $E_{2} / E_{1}$ and $h_{u} / \ell_{1}$ on the values of the ERR in the various $\ell_{0} / \ell_{1}$ in the case where $s / \ell_{1}=0$.

\begin{tabular}{llllll}
\hline $\boldsymbol{E}_{2} / \boldsymbol{E}_{1}$ & $h_{u} / \ell_{1}$ & $\ell_{0} / 2 \ell_{1}$ & & & \\
& & $\mathbf{0 . 0 4}$ & $\mathbf{0 . 1 0}$ & $\mathbf{0 . 1 5}$ & $\mathbf{0 . 2 5}$ \\
\hline 0.5 & 0.10 & 0.3484 & 1.8129 & 4.9865 & 20.9955 \\
& 0.0833 & 0.3547 & 1.9037 & 5.3128 & 22.9299 \\
& 0.0666 & 0.3774 & 2.2272 & 6.5064 & 30.2514 \\
& 0.05 & 0.4338 & 3.0427 & 9.5460 & 49.4419 \\
1 & 0.10 & 0.2179 & 1.1878 & 3.3233 & 14.4063 \\
& 0.0833 & 0.2220 & 1.2492 & 3.5439 & 15.7873 \\
& 0.0666 & 0.2371 & 1.4714 & 4.3529 & 20.9374 \\
& 0.05 & 0.2750 & 2.0241 & 6.4620 & 34.5191 \\
5 & 0.10 & 0.1072 & 0.6028 & 1.5980 & 6.2118 \\
& 0.0833 & 0.1099 & 0.6307 & 1.6935 & 6.7353 \\
& 0.0666 & 0.1192 & 0.7307 & 2.0411 & 8.6579 \\
& 0.05 & 0.1404 & 0.9767 & 2.9222 & 13.7326 \\
\hline
\end{tabular}

influence of the material anisotropy on the ERR increases with a decrease in the values of the ratio $E_{2} / E_{1}$.

Table 3 illustrates the influence of the ratios $\ell_{3} / \ell_{1}$ on the ERR for various values of $E_{2} / E_{1}$ obtained in the case where $\ell_{0} / 2 \ell_{1}=0.15, s / \ell_{1}=0$ and $h_{u} / \ell_{1}=0.1$ (i.e. $h_{u}=h / 2$ ). As can be seen, the values of the ERR monotonically increase with $\ell_{3} / \ell_{1}$. This effect becomes more pronounced with decreasing of the ratio $E_{2} / E_{1}$.

Table 4 shows the effect of the crack's location and crack length on the ERR for various values of $E_{2} / E_{1}$ in the case where $s / \ell_{1}=0$. It can be concluded that the values of the ERR increase as the crack location approaches the upper face plane of the plate i.e. as the values of $h_{u} / \ell_{1}$ decrease. This effect becomes significant with crack length, i.e. $\ell_{0} / 2 \ell_{1}$.

\section{Conclusion}

In the current paper, the ERR in the band crack's front which is contained in the thick rectangular plate made of multilayered composites is investigated by employing 3D FEM. The opening mode case which is considered is based on the numerical results of the influence of the plate material anisotropy and crack's location geometry on the ERR, which are presented and discussed. At the same time, the approach used here can be applied to the related materials considered in works $[16,17]$. 
Conflict of interest: Authors state no conflict of interest.

\section{References}

[1] Akbarov S.D., Turan A., Mathematical modelling and the study of the influence of initial stresses on the SIF and ERR at the crack tips in a plate-strip of orthotropic material, Appl. Math. Model., 2009, 33, $3682-3692$.

[2] Akbarov S.D., Turan A., On the energy release rate at the crack tips in a finite pre-strained strip, CMC Comp. Mater. Cont., 2011, 24, $257-270$.

[3] Akbarov S.D., Turan A., Energy release rate in a prestressed sandwich plate-strip containing interface cracks, Mech. Compos. Mater., 2009, 45, 597-608.

[4] Akbarov S.D., Yahnioglu N., On the total electro-mechanical potential energy and energy release rate at the interface crack tips in an initially stressed sandwich plate-strip with piezoelectric face and elastic core layers, Int. J. Solids. Struct., 2016, 88-89, 119-130.

[5] Yusufoglu E., Turhan I., A mixed boundary value problem in orthotropic strip containing a crack, J. Franklin Inst., 2012, 349, 2750-2769.

[6] Wang B.L., Niraula O.P., Two collinear antiplane cracks in functionally graded magnetoelectroelastic composite materials, Mech. Compos. Mater., 2009, 45, 583-596.

[7] Mansoor M., Shahid M., Fractographic evaluation of crack initiation and growth in AL-CNTs nanocomposite fabricated by induction melting, Acta Phys. Pol. A, 2015, 128, 276-278.
[8] Ding, S.H., and Li, X., The collinear crack problem for an orthotropic functionally graded coating-substrate structure. Arch. Appl. Mech., 2014, 84, 291-307.

[9] Harvey, C.M., Wood, J.D., Wang, S., Brittle interfacial cracking between two dissimilar elastic layers: Part 1-analytical development, Compos. Struct., 2015a, 134, 1076-1086.

[10] Harvey, C.M., Wood, J.D., Wang, S., Brittle interfacial cracking between two dissimilar elastic layers: Part 2-numerical verification, Compos. Struct., 2015b, 134, 1087-1094.

[11] Oneida E.K., van der Meulen M.C.H., Ingraffea A.R., Method for calculating $\mathrm{G}, \mathrm{G}_{1}$, and $\mathrm{G}_{||}$to simulate crack growth in $2 \mathrm{D}$, multiplematerial structures Eng. Fract. Mech., 2015, 140, 106-126.

[12] Motamedi, D., Mohammadi, S., Dynamic crack propagation analysis of orthotropic media by the extended finite element method. Int. J. Fract., 2010, 16, 21-39.

[13] Zienkiewicz, O. C., Taylor, R. L., The finite element methods: basic formulation and linear problems, Vol. 1, 4th Ed., McGraw Hill, New York, 1989.

[14] Lekhnitskii S.G., Theory of elasticity of an anisotropic body, Holden Day, San Francisco, 1963.

[15] Sih G., Handbook of stress intensity factors, Lehigh University, Bethlehem, PA, 1973.

[16] Rutci A., Failure analysis of a lower wishbone, Acta Phys. Pol. A, 2015, 128, 75-77.

[17] Petrovic M., Voloder A., Flexural strength reduction in cemented carbides, Acta Phys. Pol. A, 2015, 128, 23-25. 